\title{
Machine Learning Combinatorial Frameworks for Architecture
}

\author{
${ }^{1}$ Joshua Lye, ${ }^{2}$ Alisa Andrasek \\ ${ }^{1,2}$ School of Architecture and Urban Design, Royal Melbourne Institute of Technology, \\ Melbourne, Victoria, Australia
}

\begin{abstract}
This paper investigates the application of machine learning for the simulation of larger architectural aggregations formed through the recombination of discrete components. This is primarily explored through establishing hardcoded assembly and connection logics which are used to form the framework of architectural fitness conditions for machine learning models. The key machine learning models researched are a combination of the deep reinforcement learning algorithm proximal policy optimization (PPO) and Generative Adversarial Imitation Learning (GAIL) in the Unity Machine Learning Agent asset toolkit. The goal of applying these machine learning models is to train the agent behaviours (discrete components) to learn specific logics of connection. In order to achieve assembled architectural 'states that allow for spatial habitation through the process of simulation.
\end{abstract}

Keywords: High Resolution, Automated Assembly, Simulation, Combinatorics, Machine Learning, Logics of Connection, Discrete Elements.

\section{Introduction}

Picking up upon the ideas of Henry Ford's assembly line and early precedents of prefabrication in architecture, notions of architectural production through a kit of parts or building blocks is seemingly not a new idea. Historically prefabrication challenged the traditional practices of construction through its ability to engage with the concerns of rapidly growing populations that demanded a faster and cheaper alternative. At the height of prefabrications success mainly due to the necessity of the post-world war era, business models such as Alladin homes or Sear kit homes began to emerge to address the struggling housing market of the time and offer an alternative affordable housing model to the masses. Owners could pick from a plethora of homes and styles, marketed to them in a telephone directory style catalogue that was ready to ship and construct. However, to the detriment of the prefabrication concept this boom in popularity and necessity meant that over time a certain attitude towards prefabrication formed. Prefabrication began to be seen as a homogenous and largely standardized model of architecture. If we trace the project lineage of most prominent architects throughout the discourse, most have tried their hand at the prefabricated model in some shape or other. The allure of prefabrication is high, yet its recent success is stagnant. Amongst the homogeneity of mass housing, some architects such as Moshe Safdie, Jean Prouve, Shigeru Ban and others begin to offer richer prefabrication models as a driver for design thinking rather than a pure method of construction.

Fast track to the present, the way we design, and construct buildings is lagging behind evolving inhabitation's rapidly increasing quantitative and qualitative demands. Greater integration of big data and AI in design processes, as well as automation in construction is needed to enable us to design for complex environments. Inherently architecture has always been linked to the tools and materials available at the time. New ways of conceiving design have often led to a disconnect with current construction technologies. Highly complex architectural designs often require bespoke construction or fabrication methods which are 
overly expensive or complicated. In the current landscape, prefabrication technologies are advancing, with companies such as IKEA's (Bloklok Housing) and Katerra (Aiya) that can now mass-produce at a highly automated and customizable level thanks to advances in fabrication technology. Prefabricated architectural outputs, however, still adhere to past preconceived models of what architecture should be, failing to take opportunities for possible expansion in both formal expression and heightened performance.

In this paper, we are proposing the increase in resolution of discrete building blocks within prefabrication in order to uncover new architectural possibilities, through both computational design and automated construction. In this scenario, syntax of architecture emerges through recombination of discrete building blocks that can be standardized, customizable, and reconfigurable. The platform for architecture subsequently occurs through the interaction of a lager population of building blocks and higher resolution complex aggregates, responding and adapting to contingent data sets. By its nature these systems are deeply computational since universality and simplicity of discrete building blocks allows for virtually infinite combinatorics and emergence of complex tectonic states through the collective action of underlying elements. Since these discrete elements already carry information about underlying constraints of fabrication, it allows for higher articulation without jeopardizing the constructability or flexibility of the model.

The research displayed in this paper builds upon Andrasek's work and design methodologies, particularly concepts of high-resolution architecture and methodologies of working through a process of design search within information-rich design environments. Those environments are built upon a synthesis of multitude of algorithmic procedures and big data sources. Andrasek's Bloom Games project in collaboration with architect Jose Sanchez establishes a lineage of projects that are based on principles of discreteness and redundancy. Commissioned by the office of the Mayor of London, as part of the WONDER series of architectural installations to celebrate the 2012 Olympics and Paralympics. Bloom is conceptualized as an urban toy, the physical assembly of basic components that are aggregated to form larger structures showcases the infinite possibilities of a basic rule system that is defined by the pure logics of connection alone and enriched by the contingency of creative human play.

\subsection{Disrupted Distributed - Computing Infinite States}

Bloom and its predecessors that Andrasek has been developing since 2001, all shared the basic principles of generating complexity out of simple discrete building blocks, where very small input would generate very complex and rich output, following one of the basic principles of complexity. However, those systems always relied on randomness coming from a human assembly of discrete elements, and since contingency is incomputable, it was impossible to simulate complexity of connection logics through the digital simulation. This problem was clearly outlined in a research studio at RMIT in 2019 entitled 'Disrupted Distributed' which resulted in a 1:1 in situ physically assembled wall (Figure 1.1) and design proposals for an artist residence. 
Joshua Lye, Alisa Andrasek

Machine Learning Combinatorial Frameworks for Architecture

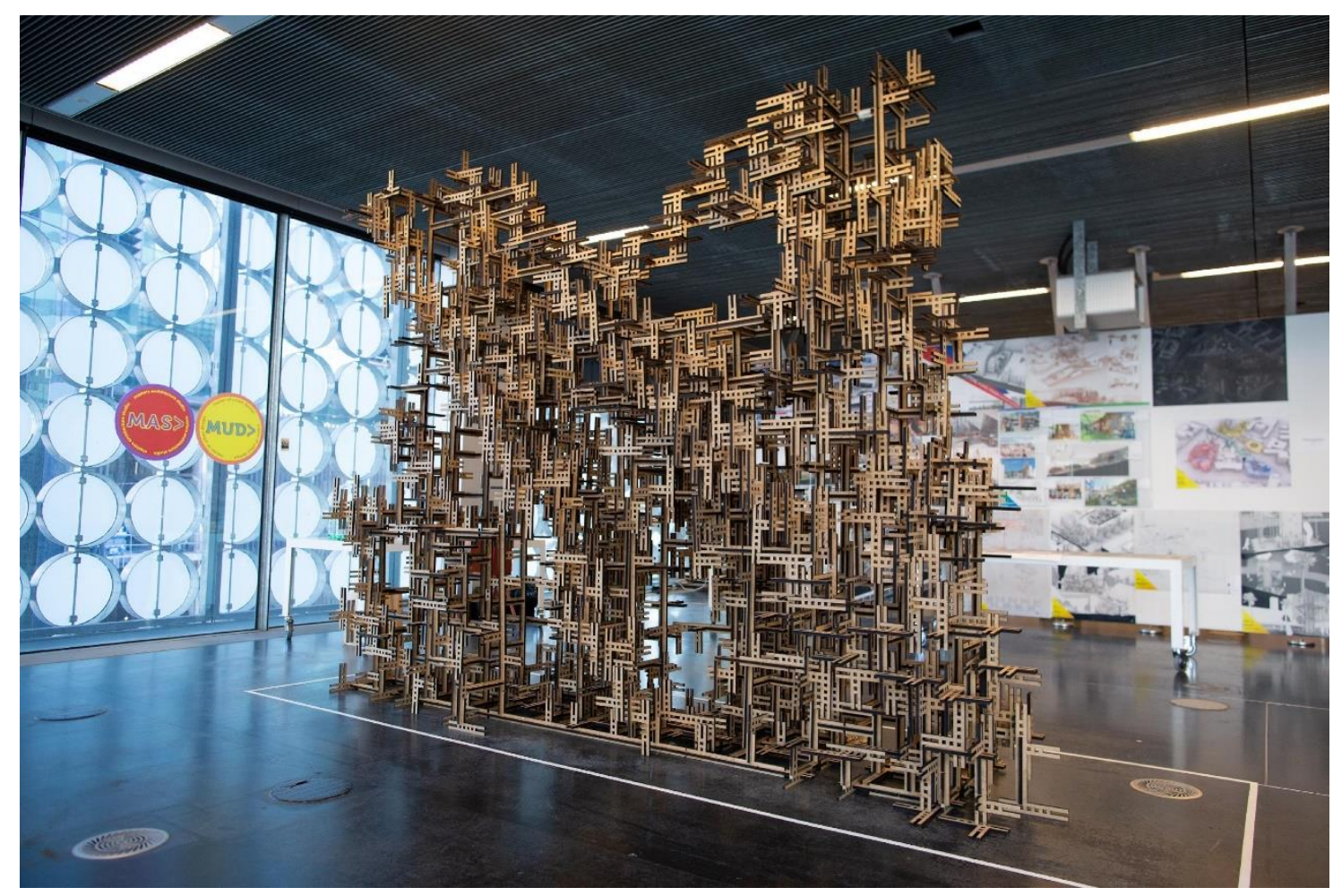

Figure 1: 1:1In Situ Wall - Photogr:aphy Adesina, TemiTope - RMIT University

In this project a universal component is designed (Figure 1.2) with three main connection joints and six interior hole joints. The geometry of components is basic due to its fabrication being constrained to rigid, laser-cut elements, however, the connection logics posed multiple design and computational challenges. The first being that the possible connection logics of this universal component produced 78 unique states (Fig 1.3) for a single connection. To address this, an agent-based algorithm was deployed, capturing the component's connection logic. This produced data maps for zoning, density and directionality of components in the assembly of larger architectural aggregations. Fitness conditions were determined based on qualitative aspects such as organizational properties of space or emergent pattern directionality. Different rule-based approaches were explored to constrain the assembly logics, such as the formation of super states that defined areas with increased structural capacity or identifying redundancy within the system where aggregated states would result in collisions.

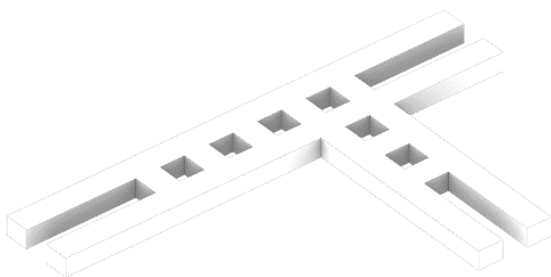

Figure 2: Universal Component 


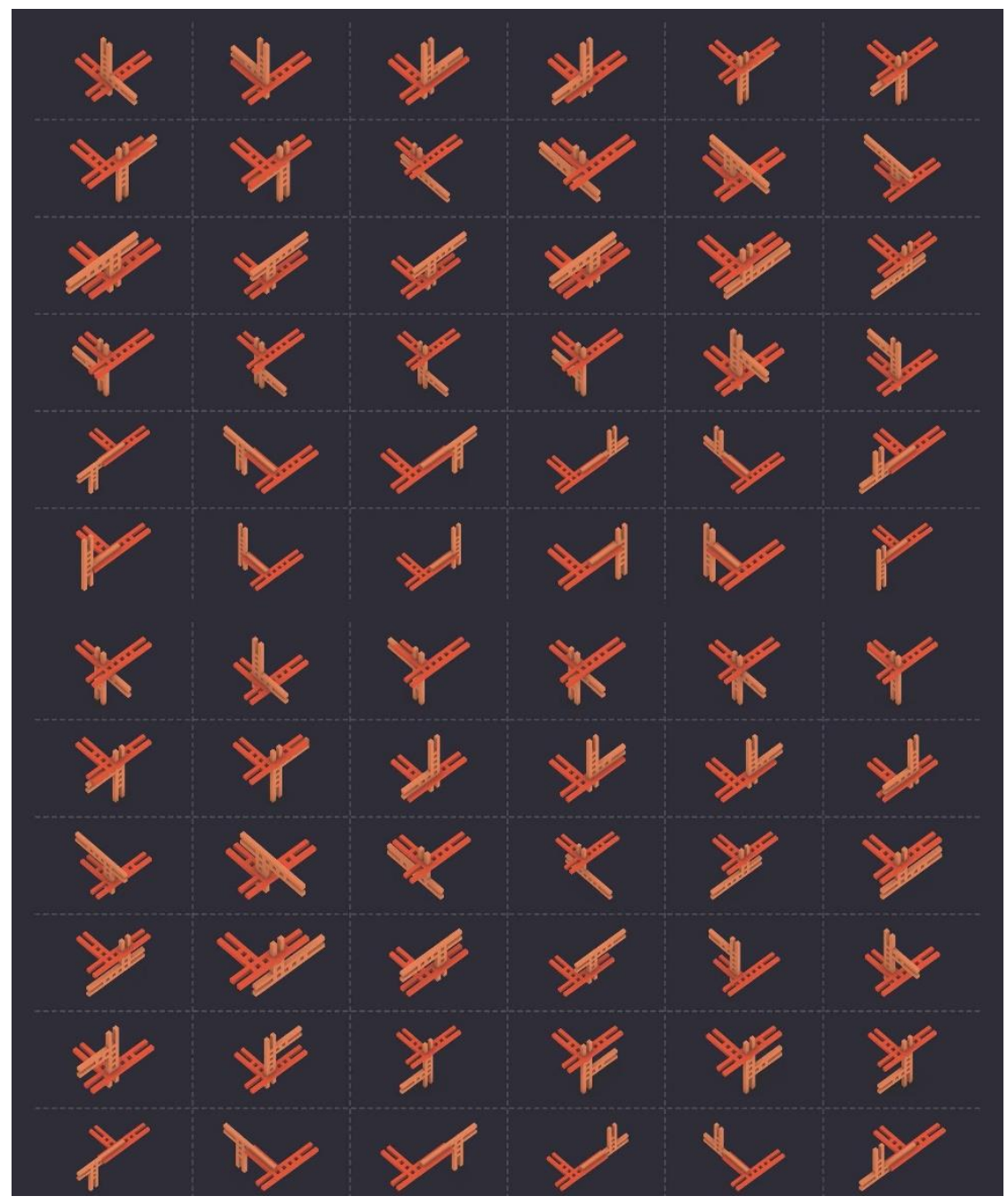

Figure 3: 78 Possible States - Image Credit Adesina Isobel Moy, Temitope Adesina, Neal Kaldor, Benjaman Verijl, Boby Subagyo Putro, Sammy Kudret, Simon Haidar, Jarrod Allen, Qingqin Yang, Tongyang Xu - RMIT University

Physical prototyping provided a useful feedback tool in the design process as it allowed for a quicker understanding of the possible outcomes that different connection states produced. When undertaking the assembly of the $1: 1$ in situ wall, hololens technology was used to view a 3d mapping of components seen in augmented reality to help guide the physical assembly process. In AR, colour was used to map different field properties such as characteristic density or dominant directionality of the components and resultant pattern. However, due to a big data problem and impossibility of simulating the exact component states at each point in the structure, assembly was relying on local improvisation based on student's experience and iterative process. Like in Bloom, assembly was contingent on randomness coming from creative human play. Intuition, built up of experience and decision making in actual physical prototyping provided a quicker way to iterate, detect redundancies and identify usable states. In simulation, trying to populate those fields with exact positions of the components was virtually impossible due to a big data problem that occurred through its underlying logics. As in Borges' story, at every fork in the road there are innumerable possible futures in which the structure could unfold. With the longer view towards automated construction, it becomes important to resolve simulation for such an assembly system.

The challenges faced in the 'Disrupted Distributed' project formed the basis for further research in integrating machine learning into the simulation environment for finding complex and high-performance aggregations based on discrete components and their underlying connection states. 


\section{Architectural Fitness Conditions - Combinatorics Design Search}

Reinforcement learning provided a suitable ML model due to its ability to make continuous sequential decisions based on observations measured from its environment. Coupled with this is the reward signal component of reinforcement learning, which acts to indicate to the agent how well it is completing the task. This is predominantly achieved by establishing a variety of architectural fitness conditions that aid the agent's assembly behaviour during the learning process as an evaluation metric to classify positive and negative outcomes to the agents.

In the initial stages, in order to minimize the number of variables, discrete elements were distilled down to abstract geometrical forms such as elongated rectangular sticks or basic voxelbased geometries. The connection logics investigated are based on face to face proximity connection points rather than the complex connection joints seen in the previous 'Distributed Disrupted' project. Even with basic components that are limited in the number and kind of connection points, the virtually infinite possible states when assembling means that not all assembled states achieve structurally stable or inhabitable spatial outcomes when assembled based on pure logics of connection. Machine learning agents are given samples of components that are assembled at the beginning of the simulation. This acts as initializer for the assembly process and provides the initial fitness condition for the agents to adhere to (Fig,1.4.). The agents are given logics of how to move in $x, y, z$ direction and are given negative rewards for intersecting geometry in the reinforcement learning process. 
Joshua Lye, Alisa Andrasek

Machine Learning Combinatorial Frameworks for Architecture
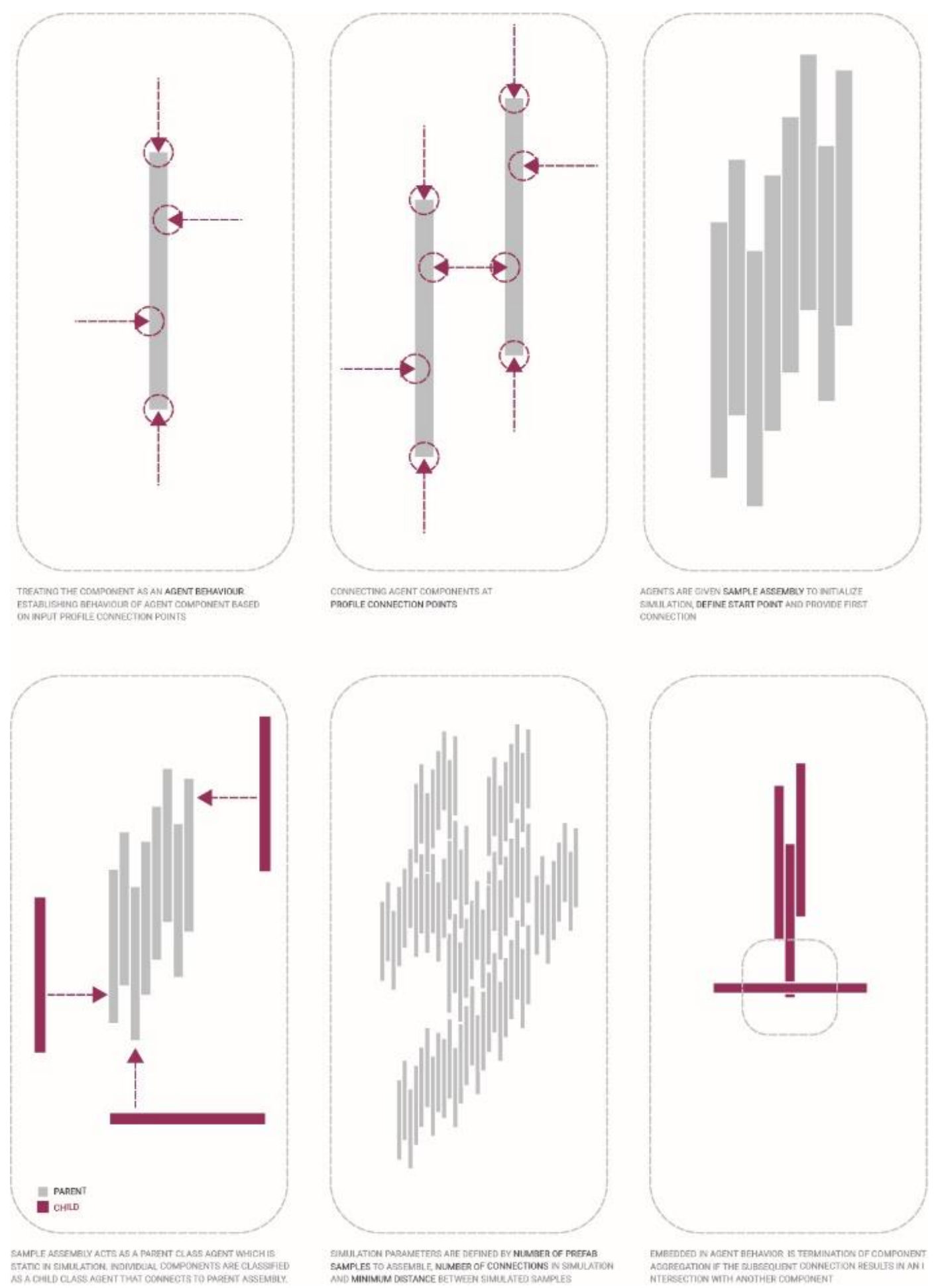

Figure 4: Connection Logics

Initial studies from the design search would often result in spherical arrangements, components intersections and dense spatial clustering (Figure1.5). The agent would try to fill all available space in the aggregation due inputting a large number of possible connections and a high number of components being assembled. This showed that there was a certain threshold in the number of components and connection points in the assembly simulation that dictated when spherical dense clustering would occur. This was subsequently communicated to agents as a negative reward due to the behaviours of the assembly achieving a spatial 
clustering that didn't meet a space filling criteria that was fit for human inhabitation. To combat this, greater emphasis was placed on the initial fitness conditions of the sample assembly. Further implementation of fitness conditions that privileged horizontal and vertical datums were established to achieve more consistent spatial clusters.

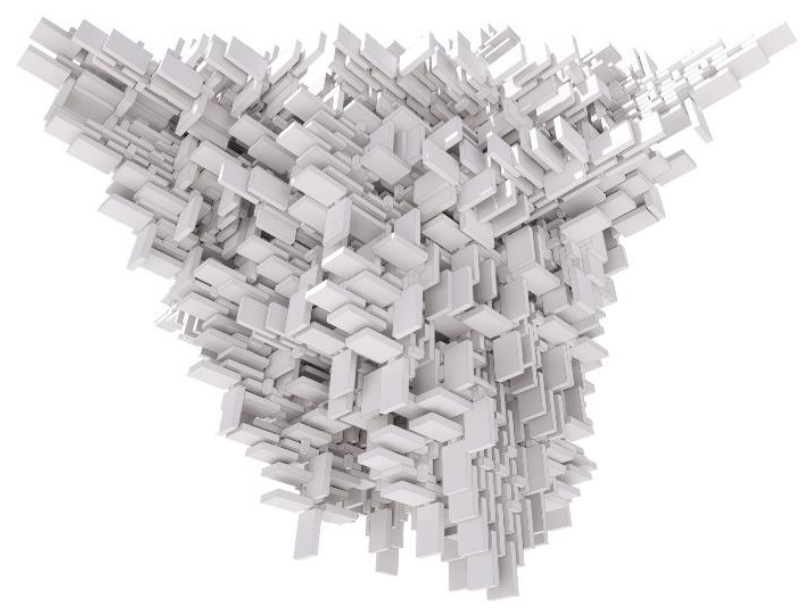

Figure 5: Initial Simulation Study

As the design search progressed spatial sequences began to emerge through iterative limiting of components connection points and running the simulation for longer periods of time. Lower resolution components began to organize larger floor-like sequences as well as forming complex circulation paths (Figure1.6). These sequences begin to suggest that the discrete elements would cluster around certain assembled states and then distribute through the aggregation rather than consistently assembling similar spatial spaces repetitively. This was a positive result, showing that the discrete elements were finding different states through the emergent combinatorics in the assembly process resulting in unique instances of architectural fabric. However, elements were still appearing to assemble in a spherical field which was impart due to the relationship between the provided sample assembly and the agent's behaviours movement to continually assemble outward from the initial assembly given. 


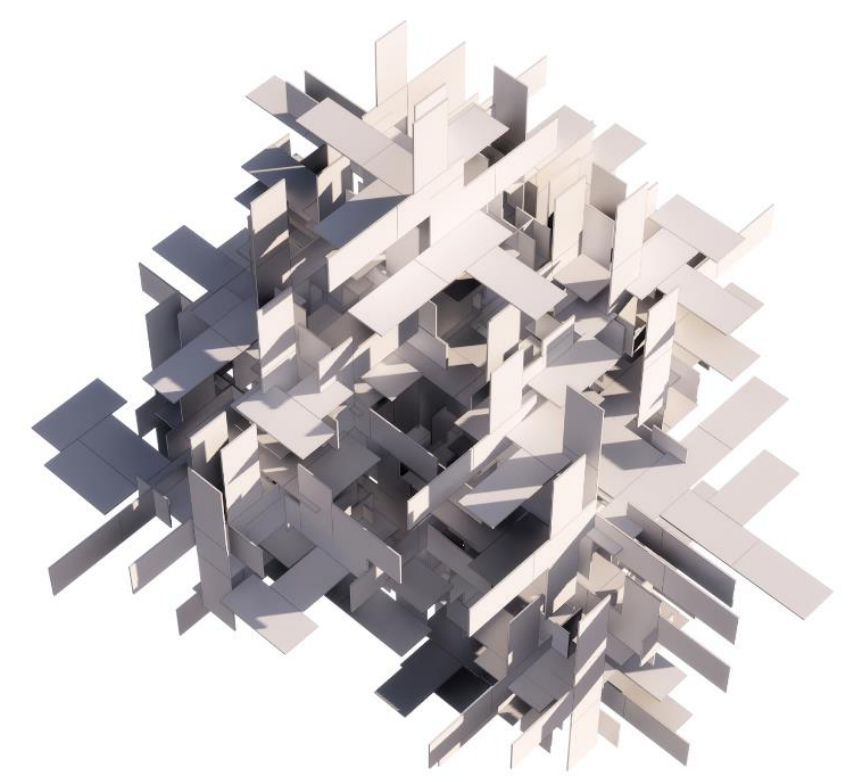

Figure 6: Lower resolution platform study

Progressing further into the design search, high resolution and multiple components were integrated as a case study to understand the algorithms capacity to deal with multiple sets of geometrical and connection data. Additionally, the simulation assembly was constrained to chunks of architectural space that approximated the scale of a residential house rather than the previous larger outcomes which are linked to vertical urban neighbourhoods. In the next phase of the design search, qualities of horizontal striation are explored through the discretization of ceilings, walls, and floors. A strategy that was employed in the designing of the components was to use a universal block joint that would facilitate the next spawning longer element. This was developed through a ball joint that could facilitate multi directional movement. In this case the ball joint is conceived as a cube which allows for only five subsequent connection faces. This gave some constraints to the simulation as the elements were essentially assembling through a voxel structure. To add variation, multiple connection points were added to singular faces to allow for components to shift between connection faces in the assembly process. The outcomes (Figure 1.7) of the design search are in embryological states of architecture, fragments of spatial articulation through discrete elements. 

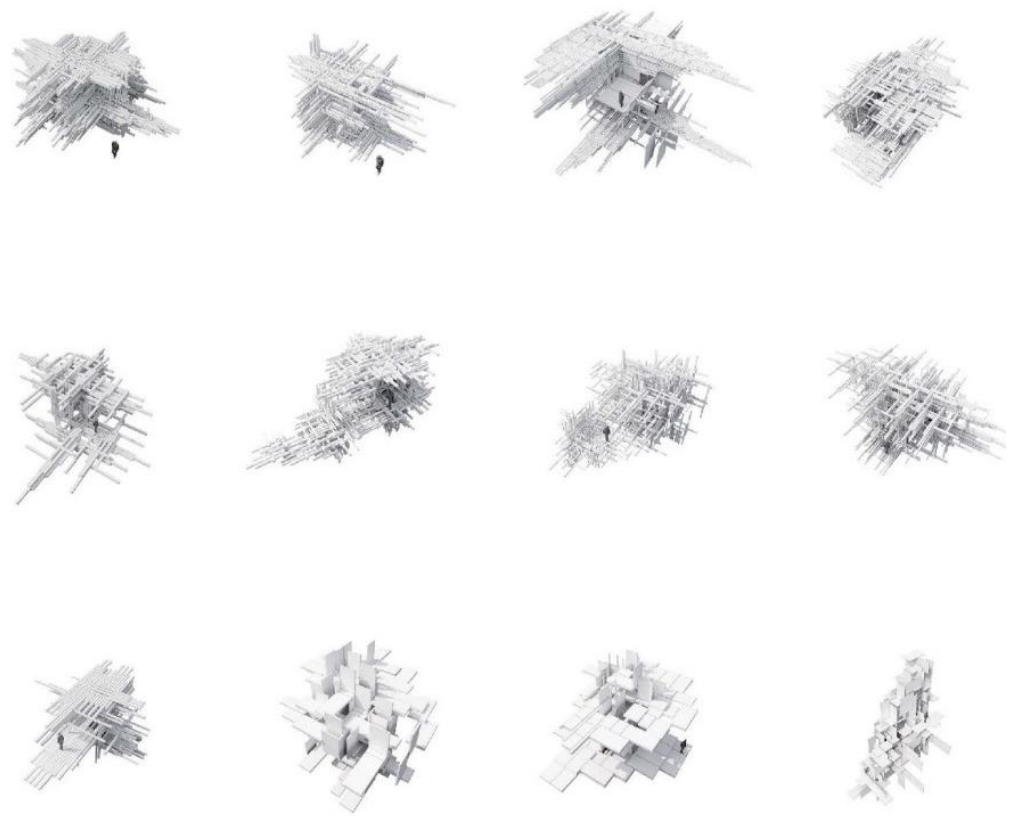

Figure 7: Lower resolution platform study

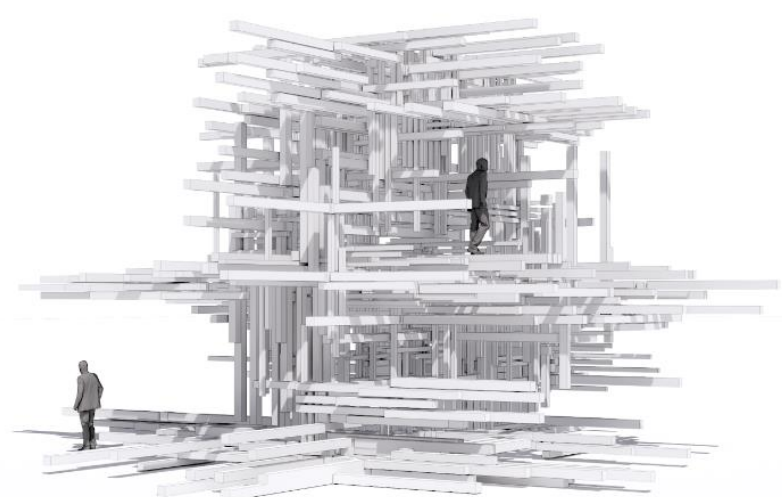

Figure 8: Assembled chunk study

\section{Conclusion and Recommendations}

This research has shown that formulating problems for reinforcement learning can be quite challenging and should be considered as a design problem in itself. Setting up an architectural framework and architecture specific fitness conditions is imperative in applying the logics of machine learning into the design process. The criteria for success and judgement of design must be translatable into the logics of the machine learning application, which is especially 
relevant in this case as it is used as a key metric to communicate to agent's how successful tasks are being completed. Ultimately the enhancement of the design process with machine learning could offer an increased level of designability of these complex interconnected systems. It needs to be adapted to information-rich design environments, where design is sculpted out of a sea of contingent data, and in a partnership with Ai cognition entirely different from our own. It will bring design of buildings and cities closer to resonating the complexity of true ecology.

\section{References}

- Andrasek, A 2018, High resolution fabric of architecture, Doctor of Philosophy (PhD), Architecture and Urban Design, RMIT University.

- Carpo, Mario. The Second Digital Turn : Design Beyond Intelligence. Cambridge, Massachusetts: MIT, 2017. Print. CrossRef

- Rifkin, Jeremy, 2019, The Green New Deal: Why the Fossil Fuel Civilization Will Collapse by 2028, and the Bold Economic Plan to Save Life on Earth, Print, St. Martin's Publishing Group

- Juliani, A., Berges, V., Teng, E., Cohen, A., Harper, J., Elion, C., Goy, C., Gao, Y., Henry, H., Mattar, M., Lange, D. (2020). Unity: A General Platform for Intelligent Agents. arXiv preprint arXiv:1809.02627. https://github.com/Unity-Technologies/ml-agents. 